\title{
Emerging antibiotic resistance and plasmid dna in escherichia coli isolated from respiratory tract infections in Bangladesh
}

\begin{abstract}
The study aimed to investigate antibiotic resistance pattern and presence of plasmid DNA among Escherichia coli isolates from respiratory tract infections (RTIs). A total of 32 E.coli isolates were characterized by using selective media and biochemical tests. Kirby-Bauer disc diffusion method was used for antibiotic sensitivity testing. Plasmid DNA extraction was carried out using Bimboim and Doly method and detection was confirmed by agarose gel electrophoresis. Overall, $51 \%$ antibiotics were resistant and $46 \%$ were sensitive out of 20 tested antibiotics. Cephalosporin antibiotics (4/7) were highly resistant (76\%). Amoxicillin showed $100 \%$ resistance. The higher rate of resistance was observed for cefuroxime, ceftazidime, cephalexin, cephradine, gentamycin and nalidixic acid ranges with $75-80 \%$. E.coli showed better sensitivity to nitrofurantoin $(78 \%)$, levofloxacin $(89 \%)$ and amikacin $(100 \%)$. Amoxiclav, cefotaxime, ciprofloxacin and piperacillin/tazobactam antibiotics were sensitive to nearly $40 \%$ E.coli. $100 \%$ sensitivity observed for imipenem, meropenem, colistin, and amikacin. The plasmid profiling revealed that $92 \%$ E.coli harbored large plasmid DNA which justifies their emerging resistance to most of the antibiotics. This study concludes that nitrofurantoin, levofloxacin, cefotaxime, amoxiclav and amikacin antibiotics may be wise to prescribe to treat RTIs caused by E.coli. Further studies are recommended to explore the association between virulence factors and AR in E.coli isolated from RTIs.
\end{abstract}

Volume I Issue 2 - 2017

\author{
Shukla Promite,' Sajal Kumar Saha, ${ }^{2}$ Paritosh \\ K Roy ${ }^{3}$ \\ 'Department of Microbiology, University of Dhaka, Bangladesh \\ ${ }^{2}$ Department of General Practice, Monash University, Australia \\ ${ }^{3}$ Institute of Statistical Research and Training (ISRT), University \\ of Dhaka, Bangladesh
}

Correspondence: Sajal Kumar Saha, Department of General Practice, Monash University, Building I, 270 Ferntree Gully Road, Notting Hill VIC 3168, Australia, Tel +6I0452639559, Email sajal.saha@monash.edu

Received: October 01, 2017| Published: October 18, 2017

Keywords: antibiotic resistance, plasmid dna, e.coli, rtis, Bangladesh

Abbreviations: AR, antibiotic resistance; E.coli, escherichia coli; RTIs, respiratory tract infections

\section{Introduction}

Worldwide antibiotic resistance (AR) is a public health threat, places everyone at great risk. ${ }^{1}$ By 2050 , it is anticipated that AR will cause 10 million deaths and US \$60-100 trillion global economic loss if AR developing rate is constant as now. ${ }^{2}$ The rising bacterial resistance among Respiratory Tract Infections (RTI) has become a commonplace event. The resistance among RTIs causative pathogens of Streptococcus pneumoniae, Klebsiella pneumoniae, Pseudomonas aeruginosa and Escherichia coli contributes a significant cause of global morbidity and mortality as well. ${ }^{3-5}$ Additionally, inappropriate prescribing of antibiotics in RTIs has become a remarkable malpractice by prescribers. ${ }^{6,7}$ This practice further magnifies the emergence of antibiotic-resistant bacterial strains in addition to increased adverse effect, treatment cost, resource use and consultation with doctors. ${ }^{6,8}$ Difficulty in establishing bacterial etiology at the time of prescription in RTIs is another barrier to rational antibiotic prescribing in Bangladesh. ${ }^{9} 10$ The limited context specific surveillance data on antimicrobial sensitivity in Bangladesh further complicates antibiotic prescribing decisions by prescribers. ${ }^{10}$ The AR surveillance information has even paramount importance to develop local and national antibiotic guidelines.

Establishing updated antibiotic guidelines is pivotally important to support clinician's antibiotic prescribing decisions and to change the current irrational prescribing practices. ${ }^{1}$ And more importantly, evidence-based local antibiotic guidelines are no alternatives, because resistant microbes are increasingly being difficult to treat over time and it is being required more expensive or more toxic alternative antibiotics or higher doses, or both ${ }^{11}$ which could further facilitate the emergence and spread of antimicrobial- resistant microorganisms. In this regard, understanding of pathogen-specific (e.g. E.coli) AR pattern is worthwhile to research to inform practitioners to policymaker in planning, developing and implementing antibiotic stewardship programs in health care practices. ${ }^{1}$

From the molecular perspectives, resistance can be caused by three ways: natural bacterial resistance, genetic mutation, and transfer of resistance genes to another microorganism. ${ }^{11}$ A small DNA molecule called plasmid; physically separated from a chromosomal DNA and can replicate independently plays a major role in the mechanism of resistance development. Virulence genes and integrons are two important virulence factors carried by plasmid DNA contribute the spontaneous development of AR. ${ }^{11}$ Hence, plasmid profiling in E. coli may help to anticipate the presence of virulence factors; commonly responsible for bacterial resistance. ${ }^{11}$

E. coli species are being increasingly resistant to commonly prescribed antibiotics in many settings. ${ }^{6}$ RTI caused by $E$. coli is even not uncommon although these species are very common in UTIs. ${ }^{4}$ The investigations of E.coli resistance pattern in RTIs are understandably scarce so far. However, the presence of virulence factors in E. coli isolated from infections rather than RTIs are evidenced. ${ }^{5,6}$ This study, therefore, aimed to investigate I) AR pattern and II) presence of plasmid DNA among E.coli isolates from RTIs patients in Bangladesh. This study designed in order to inform clinicians, pharmacist and health policymakers about sensitive and resistant antibiotics to treat RTIs 
affected by E.coli and to indicate relevant future research in the light of exploring the molecular mechanism of resistance development in RTIs.

\section{Materials and methods}

Different culture media and antibiotic disks were used for characterization and antibiotic sensitivity testing of bacterial isolates. The Nutrient agar(Merck, Germany), Mueller Hinton Agar(Merck, Germany), Mueller Hinton Broth (Merck, Germany), Eosin Methylene Blue agar(Merck, Germany), MacConkey Agar (Merck, Germany), Kligler's iron agar (KIA) (Merck, Germany), Motility Indole Ornithine agar (MIO) (Merck, Germany), Simmons Citrate Agar(Merck, Germany), MR-VP (Merck, Germany), Catalase test (Merck, Germany), and commercial antibiotic discs (Merck KGaA, Darmstadt, Germany) were used in this experiment for biochemical characterization and antibiotic sensitivity of isolated E.coli species. Reagents used for plasmid DNA extraction were-

i) Solution I (Lysozyme solution) (2mg per $\mathrm{ml}$ of lysozyme, 50mM Glucose, $10 \mathrm{mM}$ EDTA, 25mM Tris $\mathrm{HCl}, \mathrm{p}^{\mathrm{H}} 8.0$ ).

ii) Solution II (Lysis Solution) $(0.08 \mathrm{~g} \mathrm{NaOH}, 9 \mathrm{ml}$ autoclaved water, $1 \mathrm{ml}$ SDS)

iii) Solution III (High salt solution) $(7.3605 \mathrm{~g}$ potassium acetate, $2.875 \mathrm{ml}$ of Glacial acetic acid were dissolved in $25 \mathrm{ml}$ of distilled water). Reagents used for Agarose Gel Electrophoresis were Agarose (Sigma Chemical CO., St Louis, MO), Trisborate EDTA buffer (TBE buffer) (Gibco-BRL), Tracking Dye (10X concentration), and Ethidium Bromide $(3 \mu \mathrm{g} / \mathrm{ml})$.

\section{Sample collection}

A total of 32 sputum samples were collected from RTI patients admitted in Medinova Medical Services LTD in Bangladesh. Demographic information for the patients was recorded with informed consent. According to Gradwohl's clinical laboratory methods and diagnosis, ${ }^{12}$ early morning sputum samples were collected and processed for analysis.

\section{Bacterial culture}

Samples were inoculated onto Nutrient agar plate followed by incubation at $37^{\circ} \mathrm{C}$ for 24 hours. The colonies inoculated on Eosin methylene Blue agar were identified as E. coli based on green metallic sheen color. Moreover, E.coli showed flat, dry, pink colonies on MacConkey plates. The colonies were sub cultured on nutrient agar to obtain pure cultures.

\section{Gram staining}

The standard Gram Staining procedures were applied to perform gram staining for all isolates. A thin smear was prepared and the slide was air dried. Heat fixation was done by passing the slide over a flame. The smear was flooded with crystal violet and was allowed to stand for 45 seconds. The crystal violet was washed away with a gentle stream of water followed by adding Grams iodine (mordant). The smear was decolorized by adding $95 \%$ alcohol to the slide and was washed away after 10 seconds. Finally, the slide was flooded with the counter stain safran in allowed to stand for 1 minute. The slide was washed, dried and made ready to be visualized under bright field microscope.

\section{Biochemical tests}

The isolates were also distinguished by using traditional biochemical tests; Kligler's Iron agar test, Motility-Indole-Ornithine test, citrate utilization test, Methyl Red- Voges Proskauer test, indole test, and catalase test.

\section{Antimicrobial sensitivity testing}

The Kirby-Bauer disk diffusion method was used to evaluate the AR pattern of 32 isolates using 20 different antibiotics. The isolates were cultured onto Mueller- Hinton agar (Merck, Germany) plates and standard antibiotic discs were placed on the surface of the inoculated agar plates. The antibiotic disks used were amoxicillin $(25 \mu \mathrm{g})$, amoxiclav $(30 \mu \mathrm{g})$, cefixime $(5 \mu \mathrm{g})$, cefotaxime $(30 \mu \mathrm{g})$, ceftazidime $(30 \mu \mathrm{g})$, ceftriaxone $(30 \mu \mathrm{g})$, cefuroxime $(30 \mu \mathrm{g})$, cephalexin $(30 \mu \mathrm{g})$, cephradine $(15 \mu \mathrm{g})$, ciprofloxacin $(5 \mu \mathrm{g})$, colistin $(15 \mu \mathrm{g})$, gentamycin $(30 \mu \mathrm{g})$, amikacin $(30 \mu \mathrm{g})$, imipenem $(10 \mu \mathrm{g})$, meropenem $(10 \mu \mathrm{g})$, cotrimoxazole $(30 \mu \mathrm{g})$, piperacillintazobactam $(30-10 \mu \mathrm{g})$, nitrofurantoin $(300 \mu \mathrm{g})$, nalidixic acid $(30 \mu \mathrm{g})$, levofloxacin $(5 \mu \mathrm{g})$ (Merck KGaA, Darmstadt, Germany) per disc. The plates were observed for the zone of inhibition after 24 hours. Antibiotic sensitivity of E.coli isolates was determined by measuring the diameter of each zone of inhibition following a standard chart and a guideline of Fluka-Sigma- Aldrich, 2015. ${ }^{13}$

\section{Plasmid profile analysis}

Plasmid DNA isolation was carried out by Bimboim and Doly (1979) method. ${ }^{14}$ One loopful of fresh culture was added to each McCartney bottle contained $5 \mathrm{ml}$ nutrient broth. $1.0 \mathrm{ml}$ of overnight culture was transferred to a $1.5 \mathrm{ml}$ eppendorf tube and was centrifuged at $13,000 \mathrm{rpm}$ for 10 minutes using a micro Centaur (D-7200) microcentrifuge. The supernatant was discarded. The pellet was thoroughly suspended in $100 \mu$ l of Solution I and was kept at room temperature for 10 minutes. $200 \mu 1$ of Solution II was added and mixed gently. It was kept on ice for 5 minutes. $150 \mu 1$ of solution III (ice cold) was added and vortex mixed. The tube was kept on ice for 5 minutes. The mixture was centrifuged for 10 minutes at $13,000 \mathrm{rpm}$ to get the pellet of chromosomal DNA. About $450 \mu 1$ of the clear supernatant containing the plasmid DNA was taken. Two volume $(900 \mu 1)$ of ice-cold ethanol $(95 \%)$ was added to each tube. It was kept at room temperature for 2 minutes for DNA precipitation. The precipitated DNA was collected by centrifugation at 13,000rpm for 10 minutes. The supernatant was discarded. The pellet was air dried at room temperature. The dried DNA was dissolved in $50 \mu 1$ of TE buffer.

\section{Agarose gel electrophoresis of plasmid DNA}

Electrophoresis was carried out with horizontal agarose gel. Agarose $(0.8 \%)$ was dissolved in $1 \mathrm{X}$ TBE electrophoresis buffer and $3 \mu \mathrm{g} / \mathrm{ml}$ of Ethidium Bromide was added. The gel was placed in an electrophoresis chamber and submerged with $1 \mathrm{X}$ TBE buffer. Then $2 \mu 1$ of loading dye was added to $10 \mu 1$ of each sample was mixed well and total $12 \mu 1$ samples were loaded into each well. A $1 \mathrm{kbp}$ molecular marker was used as a molecular weight standard and $7 \mu \mathrm{l}$ of the marker was loaded into the gel well. Electrophoresis was carried out at 70volts. The gel was then viewed on Gel Doc (Bio-Rad). The molecular weight was determined on the basis of its mobility and was compared with the mobility of the known molecular weight marker. ${ }^{15}$

\section{Results and discussion}

AR has become a crisis issue in the $21^{\text {st }}$ century. ${ }^{1}$ Antibiotics misuse and shortages of existing effective antibiotics have magnified this crisis. ${ }^{2}$ RTIs are the most frequently occurred human infections and inappropriate use of antibiotics against these infections is globally more common and most frequent including Bangladesh. This leads to 
the rise of resistant bacterial pathogens in RTIs. The resistant strains of RTI patients are consequently increasing hospitalizations, failure of treatment and mortality. ${ }^{5}$ Not only selective pressure but also the ability of bacteria to change their genetic cassette increases their immunity against antibiotics. These two phenomena may explain the rapid changes of the modality of AR among pathogenic bacteria. ${ }^{6}$ The resistance profile and plasmid profile of E.coli isolated from RTIs are under-researched. This study, therefore, focused two aspects of AR and plasmid DNA profiling among E. coli isolates from RTIs.

\section{Antibiotic sensitivity and resistance pattern of E.coli}

The study analyzed individual antibiotic sensitivity and overall sensitivity among different classes of antibiotics. It was shown that E. coli were highly sensitive $(80-100 \%)$ to carbapenem antibiotics of meropenem, imipenem and colistin. E. coli also showed very good sensitivity against nitrofurantoin (78\%) levofloxacin (89\%) and amikacin $(100 \%)$. However, only $24 \%$ E. coli showed sensitivity to cephalosporin antibiotics on average (Figure 1). Resistance profiling showed that $100 \%$ E. coli were resistant to amoxicillin and $60-85 \%$ E. coli showed resistance against other classes of antibiotics. Each of cephalosporin antibiotics of cefuroxime, ceftriaxone, cephradine, cefixime, cephalexin, ceftazidime showed resistance with $80 \%$. Aminoglycoside antibiotics of gentamycin and amikacin were found to be resistant to $80 \%$ and $0 \%$ respectively. The sulfadrug antibiotic cotrimoxazole was resistant to $65 \%$ E.coli (Figure 1). Cefotaxime was resistant to $50 \%$ species which was comparatively better responded than other cephalosporin antibiotics. Amoxiclav was even resistant to nearly $60 \%$ E.coli (Figure 1 ). This resistance pattern was surprisingly threatening for the treatment of $E$. coli mediated RTIs.

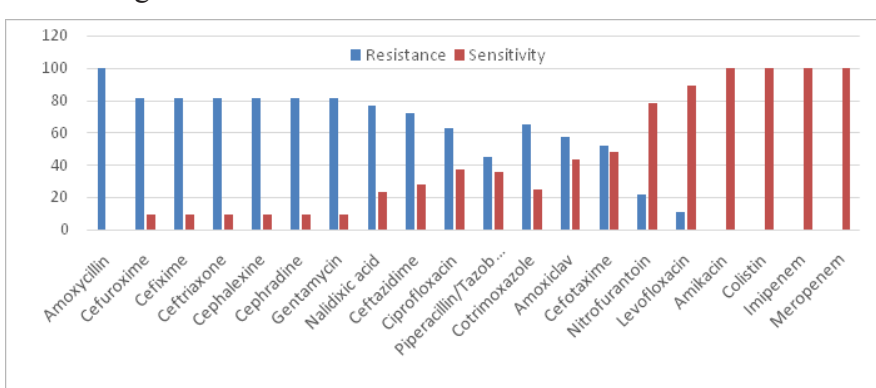

Figure I Antibiotic resistance and sensitivity pattern of E.coli.

Antibiogram reports showed that overall $46 \%$ (out of 20) antibiotics were sensitive, whereas $51 \%$ were resistant to E.coli (Table 1). However, there was significant sensitivity (17-100 \%) differences among antibiotic classes $(\mathrm{p}-$-value $<0.000$ ). Cephalosporin antibiotics were highly resistant (76\%) (Table 1). Penicillin antibiotics showed $64 \%$ resistance and aminoglycoside antibiotics resulted in $33 \%$ resistance (Table 1). Amoxiclav, cefotaxime, ciprofloxacin and piperacillin/tazobactam antibiotics were sensitive to nearly $40 \% E$. coli. $100 \%$ sensitivity observed for imipenem, meropenem, colistin, and amikacin. The higher rate of resistance $(75-80 \%)$ was observed for cefuroxime, ceftazidime, cephalexin, cephradine, gentamycin and nalidixic acid. The findings of greatest cephalosporin resistance (76\%) were similar with cephalosporin resistance data of Pakistan $(90 \%$ of 92 E.coli isolates), India (83\% of 400 E.coli isolates) and Russia (77\% of 42 E.coli isolates) (Figure 2). ${ }^{16}$ Although carbapenem antibiotics were $100 \%$ sensitive in the study isolates, however, $11 \%$ of 408 isolates in India were resistant to against them. ${ }^{16}$ The fluoroquinolones resistance was comparatively lower in Bangladesh than India (47\% vs. $84 \%$ ). The similar contradictory AR trend was also noticed for aminoglycosides antibiotics in Bangladesh and in India with $33 \%$ and $61 \%$ respectively. Although less sample size in the study makes debatable the comparison of resistance data, however rising trends of $\mathrm{AR}$ in E. coli is obvious both in Bangladesh and India. In contrast, E.coli resistance rate was significantly lower in the Australia, South Africa, United Kingdom and United States (Figure 2). ${ }^{16}$

Table I Antibiotic sensitivity and resistance pattern of RTI patient to different antibiotic classes in Bangladesh

\begin{tabular}{lll}
\hline Antibiotic class & \% Sensitivity & \% Resistance \\
\hline Penicillin & 30 & 64 \\
Cephalosporin & 24 & 76 \\
Fluoroquinolone & 53 & 47 \\
Carbapenem & 100 & 0 \\
Aminoglycosides & 67 & 33 \\
Sulfa drug & 17 & 58 \\
Average & 46 & 51 \\
P-value based on Pearson Chi ${ }^{2}$ test & 0.00 & 0.00 \\
\hline
\end{tabular}

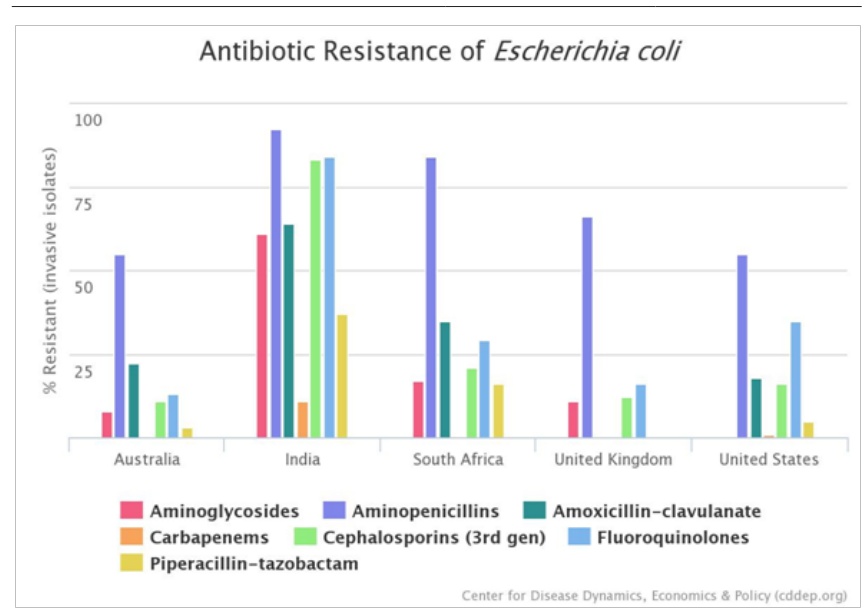

Figure 2 Antibiotic resistance of Escherichia coli in different countries. ${ }^{16}$

This higher resistance may be due to imprudent use of antibiotics in patients with RTIs. Excessive use of cephalosporin antibiotics against RTIs are common in Bangladesh and this trend may contribute to developing E.coli resistant strains. This is supported by the fact that prescribers choose cephalosporin antibiotics to avoid patients' risk of failure of response to low spectrum antibiotics. ${ }^{10}$ A systematic review ${ }^{17}$ revealed that longer duration and multiple courses of antibiotics are also associated with higher rates of resistance. Individuals prescribed an antibiotic in RTI develop bacterial resistance to that antibiotic. The greatest effect is observed in the month immediately after treatment and it may persist for up to 12 months. This effect increases the possibility of individuals' carriage of resistant organisms to first-line antibiotics and accelerates the conditions for increased use of secondline antibiotics in the community. ${ }^{17}$ This phenomenon is predictable in terms of AR pattern of E.coli revealed in the context of the study.

The results of the study have significance at the point that RTI patients still may have a better response to some fluoroquinolone and aminoglycosides antibiotics given that patients are infected with E.coli. This study emphasized that nitrofurantoin, levofloxacin, 
cefotaxime and amoxiclav antibiotics may be a wise prescription to treat RTIs caused by E.coli. The prescribers should be informed about this intervention for RTIs management especially to reduce blind prescription of $3^{\text {rd }}$ to $4^{\text {th }}$ generation cephalosporin antibiotics in Bangladesh. Moreover, it needs further investigations on large scale to validate and upgrade results of antibiotic sensitivity in RTIs. The strength of the study is an exploration of current antibiotic sensitivity pattern to RTI infection caused by E.coli which would be very informative to the prescribers in Bangladesh. This study provides a little evidence required by clinicians responsible for antibiotics prescription in RTIs to quantify the association between prescribing decisions and the occurrence of AR. It is recommended that prescribers should change their unnecessary and overprescribing attitude of broad-spectrum antibiotics in RTIs -the most important step so far to slow down the pace of rising AR in Bangladesh.

\section{Plasmid profiling of E.coli isolates}

While the clinical impact of isolating bacteria with AR warrants further research, resistance is not just a feature of the infecting organism. It is also related to the individual's bacterial gene pool. This is because plasmids carry resistance genes and which can be transferred between commensal organisms and potential pathogens. ${ }^{18}$ The transmission of commensal organisms between individuals and overprescribing of antibiotics in many contexts frequently occur. Even a transient effect of antibiotic use on the carriage of resistant organisms by an individual could have a major impact in the population to develop an endemic level of resistance..$^{18}$ Microbes and plasmid DNA, their relationship is neither parasitic nor mutualistic. Rather, plasmids provide a mechanism for horizontal gene transfer within a microbial population under a given environmental condition. Plasmids may carry genes that provide resistance to naturally occurring antibiotics. ${ }^{19}$ This study was therefore designed to explore plasmid profile among studied E.coli isolates.

The plasmid profiling revealed that 92\% (29/32) E.coli harbored large plasmid DNA (Figure 3), which justifies their emerging resistance to most of the antibiotics. This prevalence signifies that E.coli organisms in the study context are more likely to carry virulence and integron genes to their plasmids. The existence of virulence genes of iutA, fimA, and neuC in E.coli are even cited in some studies, ${ }^{20,21,22}$ which may contribute to their rising AR. These are believed to be the important virulence factors in many infections including RTIs caused by E.coli. ${ }^{23}$ Further studies are thus recommended to explore the association of virulence factors of E.coli with AR in RTIs.

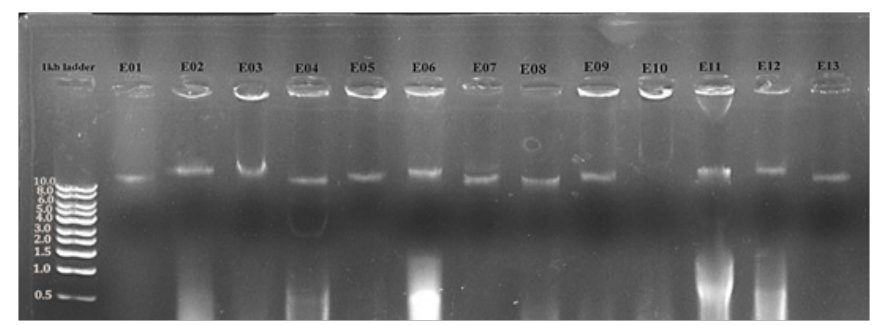

Figure 3 Plasmid profile of $E$. coli clinical isolates on $0.8 \%$ agarose gel. All the lanes $\left(E_{1}-E_{13}\right)$ except $E_{10}$ showing large plasmids of different sizes. Lane I shows I kbp DNA marker (Carl Roth).

\section{Conclusion}

The meropenem, imipenem, amikacin, colistin, and piperacillintazobactam are highest sensitive antibiotics to E.coli. Nitrofurantoin, levofloxacin and amikacin antibiotics can be better prescribing options to treat RTIs caused by E.coli. Penicillin, cephalosporin, and fluoroquinolone antibiotics are getting highly resistant to $E$. coli in Bangladesh. The presence of large plasmid DNA in E. coli justifies a risk of emerging resistance to antibiotics. The findings warrant further association studies between virulence factors and AR in E.coli isolates from RTIs.

\section{Acknowledgements}

Authors are grateful to Medinova Medical Service Ltd for giving samples of RTIs patient.

\section{Conflict of interest}

The author declares no conflict of interest.

\section{References}

1. Antimicrobial resistance: global report on surveillance. World Health Organization (WHO). 2014. 257 p.

2. El-Mahmood A, Isa H, Mohammed A, et al. Antimicrobial susceptibility of some respiratory tract pathogens to commonly used antibiotics at the Specialist Hospital, Yola, Adamawa State, Nigeria. J Clin Med Res. 2010;2(8):135-142.

3. O'Neill J. Tackling a crisis for the health and wealth of nations. The Review on Antimicrobial Resistance. 2015.

4. Jones RN, Pfaller MA. Bacterial resistance: a worldwide problem. Diagnostic microbiology and infectious disease. 1998;31(2):379-388.

5. Ball P, Baquero F, Cars O, et al. Antibiotic therapy of community respiratory tract infections: strategies for optimal outcomes and minimized resistance emergence. J Antimicrob Chemother. 2002;49(1):31-40.

6. Cantas L, Shah SQ, Cavaco LM, et al. A brief multi-disciplinary review on antimicrobial resistance in medicine and its linkage to the global environmental microbiota. Front Microbiol. 2013;4:96.

7. Martínez-González NA, Coenen S, Plate A, et al. The impact of interventions to improve the quality of prescribing and use of antibiotics in primary care patients with respiratory tract infections: a systematic review protocol. BMJ Open. 2017;7(6):e016253.

8. Baqui AH, Rahman M, Zaman K, et al. A population-based study of hospital admission incidence rate and bacterial aetiology of acute lower respiratory infections in children aged less than five years in Bangladesh. J Health Popul Nutr. 2007;25(2):179-188.

9. Alter SJ, Vidwan NK, Sobande PO, et al. Common childhood bacterial infections. Curr Probl Pediatr Adolesc Health Care. 2011;41(10):256283.

10. Rahman MS, Huda S. Antimicrobial resistance and related issues: An overview of Bangladesh situation. Bangladesh Journal of Pharmacology. 2014;9(2):218-224.

11. Alliance for the Prudent Use of Antibiotics. General background: about antibiotic resistance. Tufts University School of Medicine, 2016. 136 p.

12. Gradwohl RB, Sonnenwirth AC, Jarett L. Gradwohl's clinical laboratory methods and diagnosis. 1980.

13. Fluka-Sigma-Aldrich. Antimicrobial susceptibility test discs. 2015.

14. Bimboim HC, Doly J. A rapid alkaline extraction procedure for screening recombinant plasmid DNA. Nucleic Acids Res. 1979;7(6):1513-1523.

15. Haider K, Huq MI, Talukder KA, et al. Electropherotyping of plasmid DNA of different serotypes of Shigella flexneri isolated in Bangladesh. Epidemiol Infect. 1989;102(3):421-428. 
16. Resistance map: Antibiotic Resistance of Escherichia coli. The Centre for disease dynamics economics and policy (CDDEP). 2017.

17. Costelloe C, Metcalfe C, Lovering A, et al. Effect of antibiotic prescribing in primary care on antimicrobial resistance in individual patients: systematic review and meta-analysis. BMJ. 2010;340:c2096.

18. Chung A, Perera R, Brueggemann AB, et al. Effect of antibiotic prescribing on antibiotic resistance in individual children in primary care: prospective cohort study. BMJ. 2007;335(7617):429.

19. Wolfgang Schumann. Escherichia coli Cloning and Expression Vectors. In: Georg Lipps, editor. Plasmids: Current Research and Future Trends. UK: Caister Academic Press; 2008. p. 1-2.
20. Tokano DV, Kawaichi ME, Venâncio EJ, et al. Cloning and characterization of the iron uptake gene iutA from avian Escherichia coli. Brazilian Archives of Biology and Technology. 2008;51(3):473-482.

21. Pusz P, Bok E, Mazurek J, et al. Type 1 fimbriae in commensal Escherichia coli derived from healthy humans. Acta Biochim Pol. 2014;61(2):389-392.

22. Bekal S, Brousseau R, Masson L, et al. Rapid identification of Escherichia coli pathotypes by virulence gene detection with DNA microarrays. $J$ Clin Microbiol. 2003;41(5):2113-2125.

23. Mitra A, Palaniyandi S, Herren CD, et al. Pleiotropic roles of uvrY on biofilm formation, motility and virulencein uropathogenic Escherichia coli CFT073. PLoS One. 2013;8(2):e55492. 